\title{
АППАРАТНО-ПРОГРАММНОЕ ОБЕСПЕЧЕНИЕ АВТОМАТИЧЕСКОЙ СИСТЕМЫ ПОЗИЦИОНИРОВАНИЯ ПОДВИЖНОГО ОБЬЕКТА
}

\section{HARDWARE AND SOFTWARE \\ OF AUTOMATIC POSITIONING SYSTEM OF A MOVING OBJECT}

\section{Shamunova}

Summary. This article discusses the problems of navigation of unmanned aerial vehicles in complex dynamic environments. Navigation of an unmanned aerial vehicle in conditions of an unstable signal from satellites and dynamic obstacles on the way is an urgent task today. The existing navigation systems for unmanned aerial vehicles are mostly based on GPS navigation. Vision systems are used to navigate devices in confined spaces, where it can often be problematic to navigate using GPS. However, in conditions when the terrain on which the flight is carried out is heterogeneous with the presence of dynamic obstacles, the systems existing at the moment turn out to be untenable. The article presents the results of studies of existing navigation systems for unmanned aerial vehicles with an analysis of the advantages and disadvantages of each method.

Keywords: UAV, UAV navigation systems, vision systems, obstacle avoidance.

\author{
Шамунова Вероника Германовна \\ Аспирант, Национальный исследовательский \\ Томский государственный университет \\ evtushenko_nika@mail.ru
}

Аннотация. В данной статье рассматриваются проблемы навигации беспилотных летательных аппаратов в сложных динамических средах. Навигация беспилотного летательного аппарата в условиях нестабильного сигнала спутников и динамических препятствий на пути на сегодняшний день является актуальной задачей. Существующие системы навигации беспилотных летательных аппаратов в большинстве своем базируются на навигации посредством GPS. Для навигации устройств в замкнутом пространстве, где зачастую может быть проблематично осуществлять навигацию при помощи GPS используют системы технического зрения. Однако, в условиях, когда местность, на которой осуществляется полет является неоднородной с наличием динамических препятствий, существующие на текущий момент системы оказываются несостоятельными. В статье приведены результаты исследований существующих систем навигации для беспилотных летательных аппаратов с анализом достоинств и недостатков каждого метода.

Ключевые слова: БПЛА, системы навигации БПЛА, системы технического зрения, обход препятствий.

для работы на незнакомой местности, сильно зависят от внешних воздействий.

Очень остро сегодня стоит вопрос построения маршрутов, по которым устройства совершают полет. Не всегда местность, на которой приходится работать дрону может быть изучена с таким уровнем детализации, который позволит предусмотреть при прокладке пути все нюансы. Самой большой проблемой при построении маршрута для дрона является обход возникающих на пути препятствий. Это те препятствия, которые, возможно, не были предусмотрены разработчиком маршрута полета, или же те, которые возникают прямо во время полета (динамические препятствия).

На сегодняшний день существует не так много эффективных способов решения проблемы построения маршрута для дрона и все они так или иначе имеют существенные недостатки при их использовании для мультироторных платформ. Главной проблемой остается решение задачи обхода препятствий в динамической среде в рамках следования устройства по заданному маршруту. Поиск оптимального пути в условиях нали- 
чия препятствий на заданном маршруте сегодня является актуальной проблемой. Решение данной проблемы требует грамотного подбора аппаратно-программного комплекса для современных устройств, который бы позволил использовать БПЛА в различных условиях для выполнения поставленных задач.

Автоматические алгоритмы, используемые в БПЛА, имеющих автономный режим, в большинстве своем базируются на следующих методах [1-4]:

1. Полет по заданным GPS точкам с проверкой текущего положения устройства по показаниям бортовых датчиков;

2. Навигация БПЛА при помощи системы технического зрения (СТЗ);

3. Навигация БПЛА при помощи комплексных систем на базе GPS и систем технического зрения.

Полет по заАанным GPS точкам

с проверкой текушего положения

устройства по показаниям

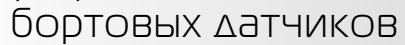

Один из самых распространенных и наиболее укоренившихся способов навигации в условиях автономной работы устройства. По данной тематике существует довольно много исследований и готовых решений, которые прошли апробацию и успешно используются на практике.

Для данного метода навигации построение любого маршрута прежде всего начинается с подробного исследования выбранной для полета местности. При исследовании должны быть учтены такие особенности, как наличие деревьев, ЛЭП, памятников, монументов и других возвышающихся над поверхностью земли объектов. На основе этих данных в дальнейшем будет подбираться оптимальная высота полета, которая так же должна зависеть от задачи, стоящей перед устройством.

Как правило, для большинства современных устройств предусматривают специализированное ПО, позволяющее задавать маршрутные точки посредством карт местности Google или Яндекс вручную, однако, существуют и решения для автоматического определения траектории. В работе [5] предложен способ построения траектории полета на основе определения оптимального пути. Такой метод может быть применен только в том случае, когда местность, на которой планируется полет заранее хорошо изучена. Для поиска оптимального пути применяется известный эвристический алгоритм $A^{*}$.

При использовании системы на базе GPS-сигнала и определения текущего местоположения путем полу- чения данных с бортовых приборов с целью корректировки маршрута, обязательно необходимо учитывать внешние воздействия на устройство, такие как ветер. Так как БПЛА, входящие в категорию, мини и микро имеют небольшие габариты, отчего ветер оказывает на них довольно большое влияние. Для решения проблемы было предложено использование нескольких контуров с ПИД-регуляторами, позволяющими выполнять необходимую корректировку управляющего воздействия на двигатели БПЛА с учетом влияния ветра [6].

Вариаций алгоритмов навигации на базе данных GPS сейчас огромное множество: это и круговой маршрут, когда на входе системе задается радиус и точка опоры, вокруг которой с заданным радиусом устройство должно летать, это и привязка БПЛА к смартфону пользователя для организации режима «слежения», когда устройство двигается за человеком. Все они применимы и могут быть довольно эффективно для различного рода задач, но только в условиях, когда есть стабильный, хороший сигнал GPS [7]. Если же устройство будет совершать полет в помещении или на территории, на которой сигналы GPS намеренно глушатся, эффективность алгоритмов, используемых данные GPS для навигации, резко и значительно снижается [8].

Еще одним существенным недостатком алгоритма является отсутствие возможности определить препятствие на пути БПЛА. Т.е. метод, основанный на использовании только систем навигации на базе GPS не способен обеспечить возможность совершения полета в сложных динамических средах.

\section{Навигация БПАА при помоши системы технического зрения (СТЗ)}

Данный метод основан на обработке изображений с подвесной камеры (или камер) мультикоптера для определения местонахождения устройства. Метод не является новым в вопросах навигации роботов в пространстве, а существует уже довольно давно и активно применяется для роботов, которые перемещаются в ограниченном пространстве.

В помещении реализовать такую навигационную систему не сложно, есть множество найденных и внедренных решений, реализующих это. Как правило, реализация выполняется с применением технологии SLAM [9]. В последнее время все больше набирает популярность использование для навигации БПЛА СТЗ совместно с искусственным интеллектом [10]. Принцип работы состоит в том, что СТ3 на базе монокулярных камер в заданный момент времени получает текущую картину сцены, которую «видит» БПЛА. Искусственный интел- 
лект выполняет обработку текущего состоянии сцены с заданным опорным изображением, которое было получено с помощью той же самой камеры, с той же самой матрицей, при построении траектории маршрута. Если текущее состояние сцены совпадает с опорным (с учетом возможной погрешности), то принимается решение о продолжении движения устройства, т.к. он следует верно.

Зачастую в средах, где использование GPS недопустимо, задействуется метод SLAM [9], который при оценке сцены использует опорные изображения, подготовленные заранее. На изображениях текущего состояния сцены SLAM выполняет поиск опорных точек, которые есть на опорном изображении. Опорные точки должны быть заранее определены путем анализа опорных изображений.

Наиболее эффективной из всех ранее представленных выше систем навигации на базе СТ3 может считаться система, принцип которой в том, что установленная на борту монокулярная камера используется не только для оценки местоположения устройства, но и для детектирования препятствий на пути робота. В качестве алгоритма для оценки сцены также предлагается SLAM, который проводит анализ сцены, на основе построенной карты глубины. Анализ карт глубины позволяет определить не только опорные точки, которые были заложены на этапе подготовки устройства к полету, но и определить наличие препятствий на пути устройства.

Таким образом, могут быть определены следующие достоинства и недостатки данного способа навигации БПЛА:

К достоинствам такого метода можно отнести следующее:

1. возможность идентифицировать динамические препятствия на пути БПЛА;

2. относительная низкая чувствительность к воздействиям из вне;

3. независимость от качества сигналов связи и прочего.

К недостаткам:

1. сложность и часто невозможность использования такой системы навигации для устройств, совершающий полет на открытой однотипной местности;

2. низкие показатели надежности и эффективности автономной работы.

Такой метод может эффективен и широко применим для различных задач в промышленных помещениях, строго ограниченных и в которых заданы маркеры маршрута. На открытой местности применимость такого метода очень ограничена, ненадежна и неэффективна без дополнительного вспомогательного оборудования и прямого участия оператора.

\section{Навигация БПАА при помоши комп^ексных систем на базе GPS и систем технического зрения}

Данный метод использует базовые принципы построения маршрута по GPS-точкам, но конструкция мультикоптера дополняется подвесом с камерой (или несколькими камерами), которая производит съемку того, что в процессе полета «видит» устройство.

Необходимость применения беспилотных летательных аппаратов в различных условиях вызвала интерес к поиску более универсальной системы, которая могла бы безотказно работать, что в условиях наличия сигнала GPS, что в условиях его отсутствия. Таким образом было предложено решение для комплексной системы, в которой СТЗ занимается задачами определения препятствий на пути устройства, а датчики на борту БПЛА позволяют следовать заданной траектории полета.

Методы навигации на базе коплексных систем базируются на следующем принципе работы: камера фиксирует с заданной периодичностью то, что «видит» устройство и передает данные системе обработки, которая может быть установлена как на дроне, так и на наземном оборудовании. Система обработки, как и в случае с навигацией с использованием только СТ3, строит карту и проверяет, нет ли на пути препятствия. Если при очередном такте обработки будет обнаружено препятствие, система обработки немедленно посылает системе управления мультикоптером управляющий сигнал для обхода препятствия. Восстановление траектории движения при этом после обхода препятствий не составляет труда, так как маршрут задан по GPS-точкам и система управления мультикоптером проверив свои текущие координаты и сверив их с заданными, понимает, что необходима корректировка, в автоматическом режиме производит ее и продолжает выдавать управляющие сигналы для выполнения строго заданного полетного плана.

Таким образом, на сегодняшний день самым эффективным способом навигации дрона в неоднородной динамической среде является применение комплексных систем, включающих в себя навигацию в пространстве при помощи GPS, бортовых датчиков и систем технического зрения. Устройства, навигация которых выполняется при помощи таких комплексных систем, могут применяться в различных сферах деятельности человека, будут менее уязвимы в средах, где сцена является динамической. 


\section{ЛИТЕРАТУРА}

1. Лоскутников А.А., Сенюшкин Н. С., Парамонов В. В. Системы автоматического управления БПЛА. // Молодой ученый. 2011. — № 9(32). - С. 56-59.

2. Лохин В.М., Манько С. В., Романов М. П., Гарцеев И.Б, Евстигнеев Д. В., Колядин К. С. Интеллектуальная система управления автономным беспилотным летательным аппаратом. // Известия Южного федерального университета. Технические науки. 2006. — C. 141-143.

3. Monocular Vision based Navigation in GPS Denied Riverine Environments / Yang J., Rao D. and others // Proceedings of AIAA Infotech at Aerospace Conference.St. Louis (USA), 2011.

4. Cesetti A., Frontoni E. A Vision-Based Guidance System for UAV Navigation and Safe Landing using Natural Landmarks / and others // Journal of Intelligent and Robotic Systems. - 2010. — № 57.—P. 233-257.

5. Kwak J., Sung Y. Autonomous UAV Flight Control for GPS-Based Navigation. // IEEE. - 2018. - $6 \mathrm{p}$.

6. Capello E., Guglieri G., Quagliotti F. A Waypoint-Based Guidance Algorithm for mini UAVs. // IFAC Proceedings Volumes. — 2013. — № 30.—P. 120-125.

7. Белоконь С.А., Золотухин Ю. Н., Котов К. Ю., Мальцев А. С., Нестеров А. А., Соболев М. А., Филиппов М. Н., Ян А. П. Управление квадрокоптером АR.DRONE при движении по заданной траектории. // Институт автоматики и электрометрии СО РАН. 2015. - C. 506-514.

8. Теодорович Н.Н., Строганова С. М., Абрамов П. С. Способы обнаружения и борьбы с малогабаритными беспилотными летательными аппаратами. // Вестник евразийской науки.—2017.— № 1. С. 1-7.

9. Engel J., Cremers D. LSD-SLAM: Large-Scale Direct Monocular SLAM. // DSO: Direct Sparse Odometry. 2016. — 67 p.

10. Степанов Д.Н. Методы и алгоритмы определения положения и ориентации беспилотного летательного аппарата с применением бортовых видеокамер.—2011.-C. 150-157.

( Шамунова Вероника Германовна ( evtushenko_nika@mail.ru ).

Журнал «Современная наука: актуальные проблемы теории и практики»

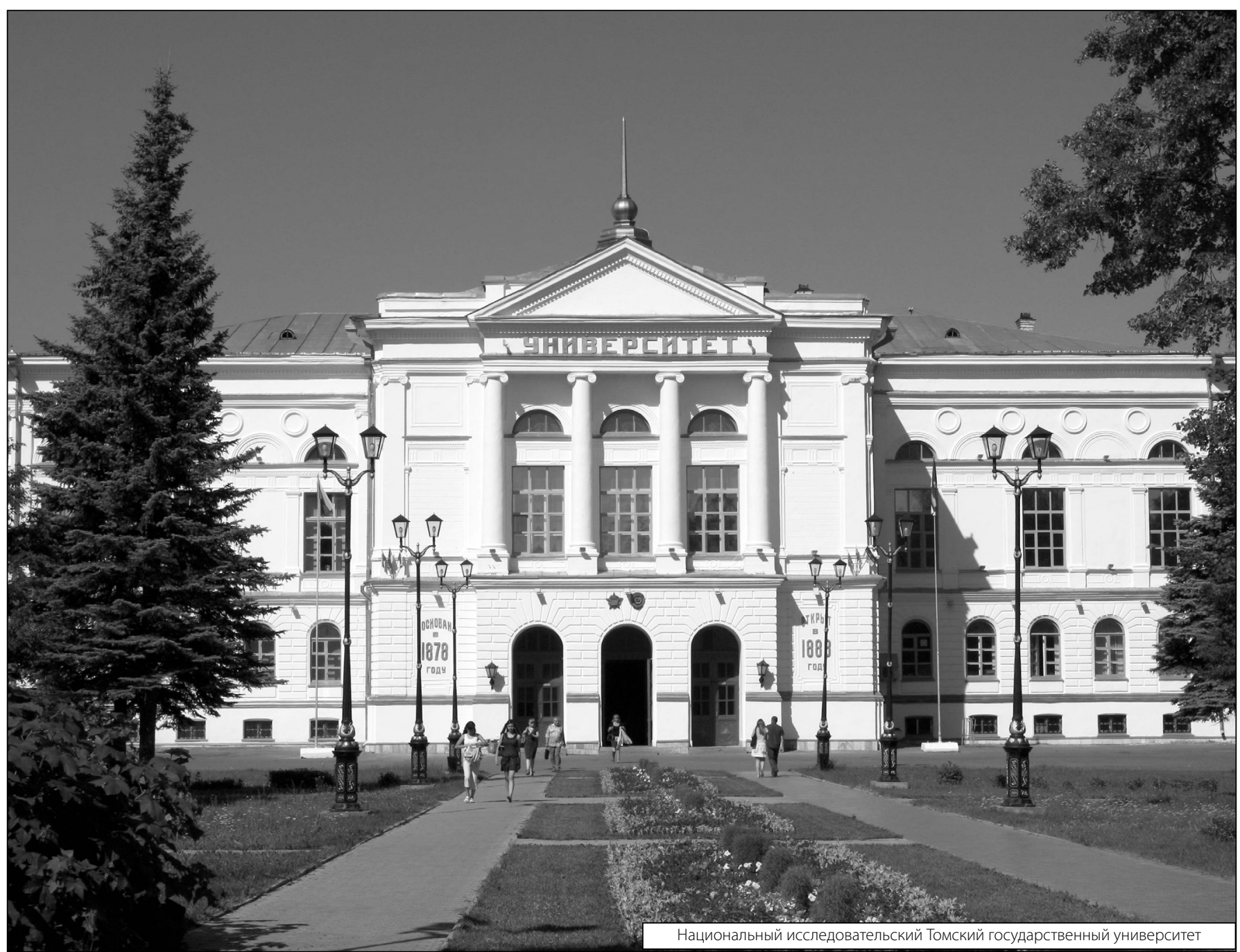

